\title{
Perfil de la condición física de mujeres adultas mayores físicamente activas
}

\section{Profile of the physical condition of physically active older adult womens}

\author{
Jairo Alejandro Fernández-Ortega ${ }^{1}$; Luz Amelia Hoyos-Cuartas ${ }^{2}$
}

\begin{abstract}
${ }^{1}$ Profesional en ciencias del deporte y la actividad física, M.Sc., Ph.D. Universidad Pedagógica Nacional. Bogotá, D.C., Colombia; e-mail: jairofdz@pedagogíca. edu.co; (i) https://orcid.org/0000-0002-6731-2645

²Licenciada en educación física, Esp.; Ph.D. Universidad Pedagógica Nacional. Bogotá, D.C., Colombia; e-mail: Ihoyos@pedagogíca.edu.co; (D) https://orcid. org/0000-0001-5362-5272
\end{abstract}

Cómo citar: Fernández-Ortega, J.A.; Hoyos-Cuartas, L.A. 2020. Perfil de la condición física de mujeres adultas mayores físicamente activas. Rev. U.D.C.A Act. \& Div. Cient. 23(2):e1274. http://doi.org/10.31910/rudca.v23.n2.2020.1724

Artículo de acceso abierto publicado por Revista U.D.C.A Actualidad \& Divulgación Científica, bajo una licencia Creative Commons CC BY-NC 4.0

Publicación oficial de la Universidad de Ciencias Aplicadas y Ambientales U.D.C.A, Institución de Educación Superior Acreditada de Alta Calidad por el Ministerio de Educación Nacional.

Recibido: Julio 4 de 2019 Aceptado: Octubre 19 de 2020 Editado por: Ingeborg Zenner de Polanía

\section{RESUMEN}

El envejecimiento saludable es una de las preocupaciones que tienen todas las naciones del mundo y está estrechamente asociado al nivel de condición física y estado nutricional. El objetivo de este estudio fue caracterizar la condición física funcional (CF) y parámetros antropométricos de mujeres adultas mayores, físicamente activas, que viven en Bogotá. Para ello, se valoró la estatura, el peso corporal, el índice de masa corporal (IMC), la velocidad de la marcha sobre 4 y 6m (VM), la velocidad media propulsiva (VMP), la fuerza máxima $(1 \mathrm{RM})$ de miembros superiores en prensa de pecho (FMS) e inferiores en extensión de piernas (FMI), la fuerza prensil (FP), la agilidad y equilibrio dinámico, la marcha estacionaria y la sentadilla, a 175 mujeres adultas mayores. Los resultados indican un decrecimiento medio gradual de la condición física entre cada uno de los grupos etarios (59-64, 65-69, 70-74, 75-79, >80 años), del 11\%, en la VM; 14\%, en la VMP; $12 \%$, para la FMS; $13 \%$, en la FMI; $12 \%$, FP; 5\%, en la agilidad y equilibrio; $9 \%$, en la marcha estacionaria y $5 \%$, en sentadillas. No se observó disminución ni en el peso corporal ni en el IMC. Las correlaciones entre las variables fueron débiles. Al interior de los grupos etarios hay gran dispersión en los valores de variables funcionales y la mayor parte de los adultos del estudio se encuentran dentro de los rangos, definidos como de menor riesgo de pérdida de la movilidad y capacidad funcional.

Palabras clave: Fuerza; Potencia; Condición física; Capacidad funcional; Adulto mayor.

\section{ABSTRACT}

Healthy aging is one of the concerns that all nations of the world have and is closely associated with the level of fitness and nutritional status. The purpose of this study was to identify the functional physical condition and body composition in a sample of 175 women between 60-83 years, physically active living in the city of Bogota. For this the valuation of the size, weight, BMI, walking speed over 4 and 6 meters (WS), mean propulsive velocity (MPV), maximum strength (1RM) of the upper body in chest press (MSU) and of 
lower body in leg station (MSL), prehensile strength (PS) through dynamometry, agility and dynamic balance, stationary walking and squats, was carried out. The results indicate a gradual average decrease in physical condition was observed between each of the age groups $((59-64 ; 65-69 ; 70-74 ; 75-79 ;>80): 11 \%$ in the WS, $14 \%$ in the MPV, $12 \%$ for the MSU, $13 \%$ in the MSL, $12 \%$ PS, $5 \%$ in agility and balance, $9 \%$ in stationary walking and $5 \%$ in squats. No decrease in body weight or BMI was observed. The correlations between the variables were weak. Within the same age groups there is a great dispersion in the values of functional variables and most of the adults in the study are within the ranges defined as having a lower risk of loss of mobility and functional capacity.

Keywords: Strength; Power; Physical fitness; Functional capacity; Older adults.

\section{INTRODUCCIÓN}

La remodelación etaria de la población emerge como un tema de preocupación, a nivel mundial, debido al incremento de la población adulta mayor, a lo largo de las últimas décadas. Según la OMS, la proporción de mayores de 60 años pasará del 11 al 22\%, entre el 2000 y 2050 y aumentará casi cuatro veces para los mayores de 80 (Organización Mundial de la Salud, 2015).

En Colombia, entre 1985 y 2014, la población mayor de 60 años registra la tasa más elevada de crecimiento: $7 \%$, en $1985 ; 10 \%$, en 2014, proyectándose al 23\%, para el 2050 (Observatorio demográfico de Amércia Latina, 2011; Fedesarrollo \& Fundación Saldarriaga Concha, 2015).

El envejecimiento es un proceso progresivo, caracterizado por el deterioro gradual acumulativo y generalizado de las funciones fisiológicas, que se puede explicar, por factores genéticos, múltiples morbilidades y factores no genéticos, como nutrición, estilo de vida y CF (Kirkwood, 2017). Conllevan a una disminución gradual de la capacidad funcional, debido entre otras causas, a la pérdida de la densidad ósea, fuerza muscular y calidad muscular, lo cual, puede predisponer el aumento en el riesgo de discapacidad (Magyari et al. 2018). Estos aspectos tienen efecto en el incremento de las caídas y fracturas, que conducen a una pérdida incapacitante de la independencia y la movilidad que va a requerir de cuidados de enfermería o atención hospitalaria (Hairi et al. 2010).

La CF representa, entre otros factores, la capacidad cardiorrespiratoria y la condición muscular de un sujeto, constituyéndola en un aspecto importante, para predecir factores de riesgo de enfermedades crónicas y mortalidad (Veronese et al. 2017). Tiene implicaciones en la calidad de vida (Rejeski \& Mihalko, 2001), debido a que se constituye en factor de riesgo para discapacidad (den Ouden et al. 2011) y para mantener un estilo de vida independiente (Mullen et al. 2012). En Colombia, se observa una prevalencia de limitaciones permanentes superior al $10 \%$ en la población mayor de 50 años. El $12,52 \%$ de los mayores de 65 años tienen limitaciones para moverse y el 3,35\%, para caminar (DANE, 2005).
Un factor clave para lograr un envejecimiento saludable es preservar la movilidad y la independencia a lo largo del tiempo, para lo cual, es necesario mantener niveles adecuados de fuerza, resistencia y agilidad, que garanticen realizar diferentes actividades de la vida diaria, como subir escaleras, levantar y transportar objetos, sentarse y pararse de una silla o caminar autónomamente (Mullen et al. 2012). Infortunadamente, la información sobre el nivel de CF necesario para mantener la independencia en los adultos mayores es limitada (Rikli \& Jones, 2013).

El propósito del estudio fue determinar la $\mathrm{CF}$ y el estado nutricional de un grupo de adultas mayores activas y, de esta forma, aportar información para construir la caracterización de la CF de los adultos mayores, en Colombia. Se buscó establecer un perfil de referencia, que permita a los profesionales que trabajan con esta población, identificar el nivel de $\mathrm{CF}$ en que se encuentran e implementar iniciativas de salud, orientadas a mejorar o mantener la $\mathrm{CF}$.

\section{MATERIALES Y MÉTODOS}

Se trata de un estudio observacional, descriptivo, de corte transversal, realizado en el laboratorio de fisiología del ejercicio de la Universidad Pedagógica Nacional, en el 2019. La muestra estuvo compuesta por 175 adultas mayores, con edades entre 60-83 años, distribuidas de la siguiente forma: 83 , entre $60-64$ años; 47 , entre 65-69; 30, entre 70-74; 11, entre 75-79 y 4, mayores de 80 años. Todas ellas eran participantes de un programa de actividad física, que accedieron a participar voluntariamente y cumplieron los criterios de inclusión:

- Adultos mayores de 60 años, menores de 85 años.

- Personas que estén participado en un programa de acondicionamiento físico en el último año, antes del estudio a realizar.

- Personas sin anemia, complicaciones renales o hepáticas.

- Diligenciar y aceptar el consentimiento informado.

Aspectos éticos. El estudio fue diseñado siguiendo las normas deontológicas reconocidas por la Declaración de Helsinki (AMM,1964) y la resolución 008430 de 1993 del Ministerio de Salud de Colombia (Ministerio de Salud y Protección Social, 1993). Las participantes fueron informadas de los detalles y objetivos del estudio, de la confidencialidad de la información y de su uso exclusivo para fines científicos y firmaron el consentimiento informado. El proyecto fue aprobado por el comité de ética de la investigación, de la Universidad Pedagógica Nacional.

Evaluación de variables. La evaluación, se realizó en cinco sesiones, con un intervalo de 72 horas entre cada una y en el siguiente orden: En la primera sesión, se adelantó la valoración médica y un tamizaje de antecedentes clínicos y estado de salud, para detección temprana de riesgo (IPAC) y nivel de actividad física, mediante el Physical Activity Readiness Questionaire (PAR-Q). En la segunda, evaluaron: el peso, la estatura, la FP y la VM; en la tercera, se aplicaron las pruebas de la batería Senior Fitness Test; en la cuarta, la fuerza máxima y, en la quinta, la potencia pico y la VMP. Días previos a las valoraciones, las participantes fueron cuidadosamente familiarizadas con el procedimiento de cada una de las pruebas. 
Antes de cada prueba, las participantes realizaron un calentamiento de 10 minutos a baja intensidad y 10min de estiramientos específicos estáticos y dinámicos. Se dejaron $3 \mathrm{~min}$ de recuperación activa entre las pruebas.

Parámetros antropométricos. Se valoró el peso corporal, utilizando una báscula electrónica (Healht Metter 599 KL, 0,50g de precisión), la estatura en posición bípeda, con un tallímetro (Detecto D52, EU) y el índice de masa corporal (IMC), se calculó con la fórmula IMC $=$ peso $(\mathrm{kg}) /$ Estatura $(\mathrm{m})^{2}$.

La clasificación nutricional, se realizó de acuerdo con la guía de la Sociedad Española de Geriatría y Gerontología (Camina-Martín et al. 2016) que, a partir del valor del IMC y de la edad, clasifica a los sujetos en: bajo peso, peso normal, sobrepeso, obesidad I, obesidad II y Obesidad III.

Fuerza máxima. La FMI y FMS, se valoraron utilizando el método de 1RM, adaptado a las características de la población (Sayers \& Gibson, 2014). Para la FMI, se utilizó una máquina de extensión de piernas, empleado en múltiples estudios (Borde et al. 2015) y para la FMS, una maquina de extensión de brazos. Para ambos casos, se inició con una carga de $5 \mathrm{~kg}$ y se realizaron cuatro repeticiones. Posteriormente, se efectuaron incrementos progresivos de $3 \mathrm{~kg}$, realizando dos repeticiones, hasta que la VMP era inferior a $0,4 \mathrm{~m} \cdot \mathrm{s}^{-}{ }^{1}$. A partir de ese momento, se realizaban incrementos de $(1 \mathrm{~kg})$, hasta cuando la participante no lograba realizar la extensión a $180^{\circ}$ y la VMP era inferior a $0,25 \mathrm{~m} \cdot \mathrm{s}^{-1}$ (Fernández \& Hoyos. 2020). La carga más elevada, se consideraba su $1 \mathrm{RM}$. El control de la VMP, se realizó con un transductor lineal de velocidad (T-FORCE Dynamic Measurement System2, Ergotech Consulting S.L., Murcia, España), con velocidad de muestreo de $1.000 \mathrm{~Hz}$ y precisión de 0,0002m) (Sanchez-Medina et al. 2014). Se dejaron 3 min de recuperación entre cada una de las series y se solicitó a las participantes que ejecutaran cada acción concéntrica, a la máxima velocidad posible.

Los valores de VMP, velocidad y potencia máxima, se determinaron a partir de los resultados obtenidos a lo largo de la evaluación de la fuerza máxima (Puthoff \& Nielsen, 2007).

Condición física. La CF, se evaluó con algunas de las pruebas de la Batería Senior Fitness Test (Rikli \& Jones, 2013), ampliamente utilizada en adultos mayores, a nivel internacional (Rikli \& Jones, 2013; Benavides et al. 2017), utilizando los protocolos establecidos en la batería. Se valoró sentadillas en 30 segundos (30-Chair Stand Test); agilidad y equilibrio dinámico (8 Foot Up And Go) y marcha estacionaria de $2 \mathrm{~min}$ (2- Minute Step Test).

Velocidad de la marcha. Se valoró la VM, debido a la asociación que tiene con la salud y riesgo de mortalidad en los adultos mayores (Lowry et al. 2011). La prueba consistió en caminar lo más rápido posible de forma cómoda, una distancia de 4 y $6 \mathrm{~m}$, de acuerdo con lo planteado por Studenski et al. (2011). Para el registro del tiempo, se utilizó el sistema de foto celdas de luz infrarroja modelo WL34R240 (Sick ® Alemania), que se ubicaron en la línea de partida y las otras, a los 4 y 6 metros. Cada participante realizó 2 ensayos, separados por un intervalo de recuperación de $3 \mathrm{~min}$ y se registró el mejor tiempo.

Fuerza isométrica. La FP de ambas manos, se evaluó con el método de dinamometría, utilizando un dinamómetro Takei (Scientific Instruments Co. Ltd, Tokyo, Japan). En posición sentado y con el brazo en un ángulo de $90^{\circ}$, la muñeca en posición neutral, manteniendo la fuerza durante 5 segundos. Se realizaron dos intentos con cada mano, con periodos de recuperación de 3 min y se registró el mejor resultado.

Análisis estadístico. Se utilizó el software SPSS Statistic ${ }^{\mathrm{TM}}$ versión 23.0. Se realizó un análisis descriptivo univariado, se calculó la media, la mediana y la desviación estándar, junto con el error estándar de la media. La comparación entre las variables, se realizó con intervalos del 95\% de confianza, se obtuvieron los comparativos entre promedios. Se aplicó la prueba T de Student, para probar hipótesis de diferencias de promedios. Previamente, se verificaron los supuestos de normalidad, homocedasticidad y suficiencia del tamaño muestral, para poder mostrar diferencias significativas, con un valor de criterio $\mathrm{p} \leq 0,05$. Finalmente, se desarrolló un análisis correlacional con el coeficiente de correlación de Pearson, para identificar relaciones significativas entre las variables de medida.

Para todos los efectos, se tomó criterio de significancia un $\mathrm{p} \leq 0,05$ y se consideró una escala apreciativa de las correlaciones, según su magnitud observada: débil, para valores $>0,40$; moderada, entre 0,41 y 0,60; fuerte, entre 0,61 y 0,80 y muy fuerte, entre 0,81 y 1,0 (Fernández \& Hoyos, 2020). Para efectos del control de sesgos (validez), el procesamiento estadístico fue desarrollado por un bioestadístico independiente del grupo de investigación.

\section{RESULTADOS Y DISCUSIÓN}

Las características de composición corporal y CF de cada uno de los grupos etarios, se presentan en la tabla 1 . No se identificaron diferencias significativas entre los grupos en el peso corporal, la estatura, el IMC y la clasificación nutricional. Cuando se observan los grupos de bajo peso, peso normal, sobrepeso u obesidad (I-II-III), al interior de cada grupo, no se presentan diferencias significativas en los resultados de las pruebas funcionales, excepto en la prueba de agilidad y equilibrio dinámico, que presentó diferencias significativas en todos los grupos etarios $(\mathrm{p}<0,05)$. El 68\% de las participantes se encontraron entre sobrepeso y obesidad tipo III (Tabla 2).

En cuanto a la CF, se observaron diferencias significativas entre los grupos etarios $(\mathrm{p}<0,001)$, en los valores medios de FP, agilidad y equilibrio dinámico, marcha estacionaria, número de sentadillas, VM sobre 4 y 6m, VMP, FMMS y FMMI; sin embargo, cuando se revisa de forma desagregada esta diferencia deja de ser significativa entre el grupo de 70-74 y los grupos 75-79 y $>80$, para las mismas variables, comportamiento que se podría deber al tamaño reducido de la muestra, de estos dos grupos. 
Tabla 1. Características de composición corporal y condición física.

\begin{tabular}{|c|c|c|c|c|c|c|c|c|c|}
\hline \multirow{3}{*}{ Variables } & $60-64$ & \multirow{3}{*}{$\Delta \%$} & $65-69$ & \multirow{3}{*}{$\Delta \%$} & $70-74$ & \multirow{3}{*}{$\Delta \%$} & $75-79$ & \multirow{3}{*}{$\Delta \%$} & $>80$ \\
\hline & $\mathrm{n}=79$ & & $\mathrm{n}=45$ & & $\mathrm{n}=29$ & & $\mathrm{n}=10$ & & $\mathrm{n}=4$ \\
\hline & Media & & Media & & Media & & Media & & Media \\
\hline Estatura $(\mathrm{cm})$ & $152,2 \pm 5,8$ & 0 & $152,8 \pm 5,1$ & 1 & $151,5 \pm 6,0$ & 1 & $150,6 \pm 5,6$ & 3 & $145,8 \pm 2,9$ \\
\hline Peso (kg) & $64,1 \pm 9,6$ & 2 & $62,8 \pm 9,6$ & 6 & $59,0 \pm 6,64$ & 4 & $61,3 \pm 8,8$ & 6 & $65,1 \pm 5,69$ \\
\hline $\operatorname{IMC}\left(\mathrm{kg} / \mathrm{m}^{2}\right)$ & $27,5 \pm 3,8$ & 2 & $26,9 \pm 4,0$ & 4 & $25,7 \pm 2,61$ & 6 & $27,3 \pm 5,3$ & 12 & $30,6 \pm 2,48$ \\
\hline Fuerza prensil (kg) & $25,0 \pm 3,0$ & 12 & $22,0 \pm 2,3$ & 9 & $19,9 \pm 2,96$ & 10 & $18,0 \pm 1,1$ & 15 & $15,3 \pm 2,63$ \\
\hline Agilidad equilibrio dinámico (s) & $5,2 \pm 0,79$ & 3 & $5,4 \pm 1,0$ & 7 & $5,8 \pm 1,05$ & 7 & $6,2 \pm 0,73$ & 3 & $6,4 \pm 2,11$ \\
\hline Marcha estacionaria (rep) & $95,1 \pm 13,5$ & 5 & $90,0 \pm 17,4$ & 6 & $84,4 \pm 15,9$ & 14 & $72,4 \pm 13,7$ & 10 & $65,5 \pm 17,3$ \\
\hline Sentadillas (rep) & $17,4 \pm 3,4$ & 2 & $17,1 \pm 4,6$ & 7 & $15,9 \pm 4,07$ & 6 & $15,0 \pm 3,9$ & 7 & $14,0 \pm 3,59$ \\
\hline Velocidad marcha $4 \mathrm{~m}(\mathrm{~m} / \mathrm{s})$ & $1,52 \pm 0,32$ & 4 & $1,46 \pm 0,36$ & 7 & $1,36 \pm 0,48$ & 13 & $1,21 \pm 0,25$ & 19 & $1,01 \pm 0 ., 4$ \\
\hline Velocidad marcha $6 \mathrm{~m}(\mathrm{~m} / \mathrm{s})$ & $1,76 \pm 0,43$ & 9 & $1,61 \pm 0,54$ & 11 & $1,45 \pm 0,57$ & 12 & $1,30 \pm 0,35$ & 14 & $1,14 \pm 0,54$ \\
\hline $\begin{array}{l}\text { Vel. Media Fase Propulsiva }(\mathrm{m} \cdot \mathrm{s}- \\
\left.{ }^{1}\right)\end{array}$ & $0,53 \pm 0,07$ & 8 & $0,49 \pm 0,09$ & 14 & $0,42 \pm 0,08$ & 12 & $0,37 \pm 0,07$ & 24 & $0,28 \pm 0,053$ \\
\hline FMS (kg) & $10,9 \pm 2,32$ & 7 & $10,08 \pm 2,4$ & 11 & $8,94 \pm 2,1$ & 13 & $7,8 \pm 1,04$ & 19 & $6,35 \pm 0,8$ \\
\hline FMI (kg) & $15,6 \pm 3,26$ & 11 & $13,8 \pm 3,1$ & 12 & $12,1 \pm 3,7$ & 11 & $10,8 \pm 1,8$ & 18 & $8,85 \pm 0,6$ \\
\hline
\end{tabular}

Tabla 2. Distribución de la población según el IMC.

\begin{tabular}{|c|c|c|c|c|c|c|c|c|c|c|c|c|}
\hline \multirow{2}{*}{ Grupo etario } & \multicolumn{2}{|c|}{$59-64$} & \multicolumn{2}{|c|}{$65-69$} & \multicolumn{2}{|c|}{$70-74$} & \multicolumn{2}{|c|}{$75-79$} & \multicolumn{2}{|c|}{$>80$} & \multicolumn{2}{|c|}{ Total } \\
\hline & $\mathbf{n}$ & $\%$ & n & $\%$ & $\mathbf{n}$ & $\%$ & $\mathbf{n}$ & $\%$ & $\mathbf{n}$ & $\%$ & $\mathbf{n}$ & $\%$ \\
\hline Bajo peso & 7 & 9 & 3 & 6,8 & 3 & 10,3 & 3 & 30 & 0 & 0 & 16 & 9,7 \\
\hline Peso normal & 15 & 19,2 & 15 & 34,1 & 7 & 24,1 & 0 & 0 & 0 & 0 & 37 & 22,4 \\
\hline Sobrepeso & 39 & 50 & 13 & 29,5 & 18 & 62,1 & 4 & 40 & 2 & 50 & 76 & 46,1 \\
\hline Obesidad III & 1 & 1,3 & 0 & 0 & 0 & 0 & 0 & 0 & 0 & 0 & 1 & 0,6 \\
\hline Total & 78 & 100 & 44 & 100 & 29 & 100 & 10 & 100 & 4 & 100 & 165 & 100 \\
\hline
\end{tabular}

Se presentaron correlaciones moderadas $(\mathrm{r}=0,41$ a 0,$58 ; \mathrm{p}<0,001)$, entre la edad y las variables de $\mathrm{CF}$, excepto con la FP, que registró una correlación fuerte $(r=0,689 ; \mathrm{p}<0,001)$. En cuanto a la disminución de la CF con la edad, se observa que, entre cada grupo etario, se presenta una pérdida gradual en la capacidad en todas las variables del estudio, con un promedio de 6,8\%, entre los 64 y 68 años; 9,3\%, entre los 69 y $73 ; 10,9 \%$, entre los 74 y 78 y $14,3 \%$, entre los 79 y mayores de 80 años. La VMP es la cualidad que presenta, a lo largo de los años, la pérdida aculada más elevada, con un 58\%, seguida de la FMI, 52\%; la FMS, 50\% y VM y FP, 46\%.

En la tabla 3, se presentan los valores máximos y mínimos y la diferencia porcentual entre cada uno de los grupos. A pesar que los resultados en cada una de las pruebas presentan una distribución normal, se observan diferencias importantes al interior de los participantes de un mismo grupo, lo que dejaría a algunas de las participantes por debajo los valores de referencia para la independencia física.

En cuanto a la composición corporal, la media del IMC obtenido es similar al reportado por Hardy et al. (2013), $26 \mathrm{~kg} / \mathrm{m}^{2}$, quienes, igualmente, no identificaron correlación entre el IMC y la edad. El IMC presentó correlaciones significativas $(p<0,05)$, débiles con las variables de $\mathrm{CF}$, de la siguiente forma: $r=0,019$, con la $\mathrm{FP} ; \mathrm{r}=0,16$, con la agilidad y equilibrio dinámico; $r=0,089$, con la marcha estacionaria y $r=0,15$, con el número sentadillas. 
Tabla 3. Valores mínimos, máximos de variables de condición física.

\begin{tabular}{|c|c|c|c|c|c|c|}
\hline \multirow{2}{*}{ Variable } & Grupo & $59-64$ & $65-69$ & $70-74$ & $75-79$ & $>80$ \\
\hline & $\mathbf{N}$ & 79 & 45 & 29 & 10 & 4 \\
\hline \multirow{3}{*}{ Fuerza Prensil (kg) } & Mínimo & 20 & 18 & 13 & 16 & 13 \\
\hline & Máximo & 32 & 25,5 & 24 & 19 & 19 \\
\hline & Dif $\%$ & 60 & 42 & 85 & 19 & 46 \\
\hline \multirow{3}{*}{ Agilidad equilibrio dinámico (s) } & Mínimo & 3,6 & 4,35 & 5,52 & 6,1 & 5,63 \\
\hline & Máximo & 7,21 & 8,36 & 9,34 & 8,32 & 9,4 \\
\hline & Dif $\%$ & 100 & 92 & 69 & 36 & 67 \\
\hline \multirow{3}{*}{ Marcha estacionaria (Número repeticiones) } & Mínimo & 53 & 45 & 56 & 55 & 50 \\
\hline & Máximo & 129 & 118 & 113 & 103 & 88 \\
\hline & Dif $\%$ & 143 & 162 & 102 & 87 & 76 \\
\hline \multirow{3}{*}{ Número sentadillas (Número repeticiones) } & Mínimo & 11 & 13 & 9 & 11 & 8 \\
\hline & Máximo & 25 & 25 & 19 & 17 & 14 \\
\hline & Dif $\%$ & 127 & 92 & 111 & 55 & 75 \\
\hline Dif $\%$; diferencia porcentual & & & & & & \\
\hline
\end{tabular}

Al comparar los resultados de la CF del presente estudio, con los valores de referencia definidos por Rikli \& Jones (2013), para cada una de las pruebas, se pudo establecer: en la prueba de levantarse y sentarse de una silla, se observó en todos los grupos un mayor número de repeticiones de, aproximadamente, el 7\%, para las participantes del presente estudio, pero los valores de pérdida acumulados entre los 60 y 84 años fueron similares (20\%). En la marcha estacionaria y agilidad y equilibrio, los valores fueron inferiores en el presente estudio, entre un 5 y 3\%, respectivamente. En cuanto a la pérdida gradual de estas cualidades, se observan diferencias con los valores de referencia (Rikli \& Jones, 2013), 31\% vs 20\%, para la marcha estacionaria y $22 \%$ vs $30 \%$, en la agilidad y equilibrio. En términos generales, no se identificó mucha concordancia con los resultados de estos estudios clásicos; esto se pude explicar por las características particulares de la población de cada estudio.

La FP se ha definido como buen indicador de la fuerza muscular de un sujeto y existe suficiente evidencia epidemiológica y experimental, que la ubica como un buen predictor de riesgo de mortalidad y para identificar riesgo de limitación en la movilidad en los adultos mayores (Wu et al. 2017; Prasitsiriphon \& Pothisiri, 2018). Su disminución ha sido asociada a riesgo de desnutrición, caídas, periodos más largos de hospitalización, menor calidad de vida relacionada con la salud y mayor riesgo de discapacidad (Prasitsiriphon \& Pothisiri, 2018).

Los resultados observados en el presente estudio son ligeramente inferiores $(8 \%)$ a los reportados por Prasitsiriphon \& Pothisiri (2018), en un amplio estudio realizado en 10 países europeos; 9\%, a los señalados por Bohannon et al. (2006), en el metaanálisis de 12 estudios llevados a cabo en Estados Unidos, Canadá, Australia, Reino Unido y Suiza. Superiores en un 39\%, a los reportados por
Nofuji et al. (2016) y Ong et al. (2017), en Singapur; 14\%, a los de Seino et al. (2014), en Japón y 8\%, a los de Sallinen et al. (2010), en Finlandia.

Estas diferencias entre los resultados ya habían sido observadas en dos grandes estudios que involucraron poblaciones de diferentes países: Sage Study On Global Ageing And Adult Health (World Health Organization, 2015) y la encuesta de salud, envejecimiento y jubilación en Europa (SHARE) (Börsch-Supan, 2016), que observaron una gran variación en los valores de la FP entre los países. Las mujeres de India y de México presentaron los valores más bajos en todos los grupos de edad; por el contrario, las mujeres de Sud África reportaron, en todos los grupos de edad, valores muy superiores; por ejemplo, en el grupo de mujeres mayores de 80 años, la FP era de $34 \mathrm{~kg}$, valor que corresponde al grupo de las mujeres de 55 años de los otros países.

Esta dispersión en los valores de la FP en los diferentes estudios, incluso de un mismo país en diferentes regiones, reafirma la idea que la fuerza está determinada por factores subyacentes, como la etnia y el tipo de actividad física que realizaron los sujetos, a lo largo de la vida. Al respecto, Moy et al. (2011), en un estudio realizado en la zona rural de Pahang Malasia, reportaron diferencias étnicas en la FP. Los aborígenes tenían una fuerza de prensión significativamente menor en comparación con los malayos, chinos e indios. Estos hallazgos refuerzan la idea que la variación genética, el estado de salud y el estilo de vida podrían explicar las diferencias entre los diversos estudios y los sujetos (McEvoy \& Visscher, 2009). Estudios genéticos indican que el polimorfismo de ACTN3 puede influir en la disminución del rendimiento de ciertas cualidades físicas con el envejecimiento (Delmonico et al. 2008). 
Otro elemento que podría explicar estas diferencias en los resultados es la diversidad de protocolos e instrumentos utilizados para medir la FP; sin embargo, esta influencia sería muy aleatoria, debido a que en los estudios que involucran diversas etnias, reportan diferencias significativas entre ellas (Moy et al. 2011). En el presente estudio, al observar la diferencia entre los valores máximos y los mínimos, se identificaron, igualmente, variaciones importantes al interior de cada uno de los grupos.

A pesar que la FP es reportada en la literatura como un indicador de la fuerza de un sujeto, su correlación con las pruebas de CF en el presente estudio fue débil con agilidad y equilibrio dinámico, número de sentadillas, VM y fuerza máxima de piernas y moderada, con la marcha estacionaria y fuerza máxima de brazos.

La velocidad de marcha (VM) también ha sido definida como una medida válida, confiable, sensible y apropiada, para evaluar y monitorear el estado funcional y la salud general, razón, por la cual, algunos estudios la han designado como el "sexto signo vital" (Castell et al. 2013; Middleton et al. 2015). La VM es el resultado de una compleja interacción de múltiples estructuras y funciones corporales, como control postural proactivo y reactivo, fuerza de la musculatura de las extremidades inferiores, limitaciones articulares, capacidad aeróbica, coordinación, propiocepción, visión o, también, se puede plantear que está determinada por 6 subsistemas fisiológicos: nervioso central, nervioso periférico, perceptivo, muscular, osteoarticular y energético (Peel et al. 2013).

Los resultados de la VM obtenidos en el presente estudio son superiores a los reportados por Nofuji et al. (2016), con diferencias del 29\%, en el grupo de 65-69 años; 8\%, en el grupo de 70-74 y $11 \%$, en el de 75-79. Son cercanos a los de Studenski et al. (2011) y Peel et al. (2013), quienes reportaron valores de 1,4 y $0,98 \mathrm{~m} / \mathrm{s}$, respectivamente.

Se indica que velocidades inferiores a $0,6 \mathrm{~m} / \mathrm{s}$, en pruebas de $4 \mathrm{~m}$, significarían un elevado riesgo de hospitalización y deterioro de la función física y la salud (Studenski et al. 2011); en todos los grupos etareos del presente estudio, ese valor fue superior.

$\mathrm{Al}$ igual que en la prueba de FP existe gran variabilidad en los resultados de la VM entre los diferentes estudios, que puede ser debida a factores de orden metodológico, como: la distancia en la que se realiza la prueba, aún no se sabe con claridad la distancia adecuada, se plantea que es sobre $10 \mathrm{~m}$, pero la mayor parte de los estudios oscila entre 4-8m; la velocidad a la que se debe ejecutar la prueba (velocidad normal o rápida) o si la prueba se inicia desde una posición estática o después de algunos metros de recorrido (Bohannon et al. 1996). Otro aspecto que pudo generar esta dispersión es el año en que se realizó el estudio; la revisión realizada por Peel et al. (2013) indica un incremento de la VM en $0.003 \mathrm{~m} / \mathrm{s}$, entre las publicaciones de 1988 comparadas con las del 2011.

De forma similar a la FP, la VM presentó correlaciones débiles y moderadas con las pruebas funcionales, de fuerza y potencia. A pesar que ambas pruebas han sido catalogadas como predictores de riesgo y mortalidad, en la literatura revisada y en el presente estudio, no se reportaron correlaciones significativas entre ellas.

En lo relacionado con la VMP en la revisión bibliográfica realizada, no se identificaron estudios en adultos mayores, tampoco se conoce su relación con factores de riesgo de mortalidad o independencia. Existen trabajos con adultos jóvenes, donde se observan valores de la VMP cercanos a $1,4 \mathrm{~m} \cdot \mathrm{s}^{-1}$ en extensión de piernas. Los investigadores coinciden que el entrenamiento con este tipo de metodología puede mejorar de forma más eficiente la fuerza y potencia máxima de los adultos mayores, disminuyendo el riesgo de daño muscular y contribuyendo al aumento de la autonomía en el desempeño de las actividades diarias (Marques et al. 2013).

Puthoff \& Nielsen (2007) observaron que la potencia máxima de miembros inferiores explicaba más la varianza en las capacidades funcionales, como caminar en distancias cortas y largas, que otras medidas de fuerza. En el presente estudio, no se constató este fenómeno. Cuoco et al. (2004) identificaron asociaciones lineales significativas $(p<0,05)$, entre la fuerza y la potencia de miembros inferiores, con el tiempo de subida de la prueba de escalón, el tiempo de pararse de una silla y la VM habitual, pero las correlaciones fueron débiles.

En cuanto a la fuerza máxima evaluada, a través del método de 1RM, existen numerosos estudios que lo realizaron con extensión de piernas o prensa de piernas o con pruebas isocinéticas y los resultados se expresaron en Newton, razón, por lo cual, no es posible realizar la comparación de los resultados. Referente a la fuerza máxima de prensa de pecho son pocos los estudios que han incluido este tipo de trabajo. El estudio realizado por Ramírez-Campillo et al. (2014) reportó valores en prensa de pecho 33\% superiores a los del presente estudio.

Otro de los aspectos identificado, en el presente estudio, son las débiles o moderadas correlaciones observadas entre las variables utilizadas, a pesar que la mayoría de ellas, de forma independiente, han sido catalogadas como indicadores de riesgo de mortalidad o discapacidad en la población adulta mayor.

Del presente estudio, se pude concluir que el grupo de adultas mayores físicamente activas, en lo relacionado con la condición física, se encuentra en niveles aceptables al igual que su estado nutricional. Por otra parte, se identificó cómo éstas varían, a través de la edad.

Colombia requiere, con urgencia, la construcción de estándares de la $\mathrm{CF}$ de los adultos mayores en los diferentes rangos etarios, de tal forma que permita detectar cuándo los índices de CF se encuentran en un nivel que podría ponerlos en riesgo de pérdida prematura de independencia física e incremento en los riesgos de mortalidad. Por ello, es necesario que estudios como este se realicen en las diferentes regiones del país.

Los resultados obtenidos en el presente estudio aportan elementos metodológicos y valores para la construcción de los estándares colombianos. 
Agradecimientos. Agradecemos a las participantes del estudio, al profesor Daniel Oliveros, por su valioso apoyo y participación y a la Universidad Pedagógica Nacional, por la financiación del estudio. Conflictos de intereses: El manuscrito fue preparado y revisado con la participación de todos los autores, quienes declaramos que no existe conflicto de intereses que ponga en riesgo la validez de los resultados presentados. Financiación: Este estudio fue financiado con recursos de la Universidad Pedagógica Nacional.

\section{REFERENCIAS}

1. ASOCIACIÓN MÉDICA MUNDIAL, AMM. 1964. Declaración de Helsinki de la AMM - Principios éticos para las investigaciones médicas en seres humanos Asociación medica mundial. Disponible desde internet en: http:/ /www.wma.net/es/policies-post/declaracionde-helsinki-de-la-amm-principios-eticos-para-lasinvestigaciones-medicas-en-seres-humanos/ (con acceso 05/11/2018).

2. BENAVIDES, L.; GARCÍA, J.; FERNÁNDEZ, J.; RODRIGUES, D.; ARIZA, J. 2017. Condición física, nivel de actividad física y capacidad funcional en el adulto mayor: Instrumentos para su cuantificación. Rev. U.D.C.A Act. \& Div. Cient. 20(2):255-265.

https://doi.org/10.31910/rudca.v20.n2.2017.385

3. BOHANNON, R.; ANDREWS, A.; THOMAS, M. 1996. Walkingspeed: reference values and correlates for older adults. J. Orthop. Sports 24(2):86-90. https://doi.org/10.2519/jospt.1996.24.2.86

4. BOHANNON, R.; PEOLSSON, A.; MASSY-WESTROPP, N.; DESROSIERS, J.; BEAR- LEHMAN, J. 2006. Reference values for adult grip strength measured with a Jamar dynamometer: A descriptive meta-analysis. Fisioterapia. $92(1): 11-15$.

5. BORDE, R.; HORTOBAGYI, T.; GRANACHER, U. 2015. Dose-Response Relationships of Resistance Training in Healthy Old Adults: A Systematic Review and MetaAnalysis. Sports Med. 45(12):1693-1720. https://doi.org/10.1007/s40279-015-0385-9

6. BÖRSCH-SUPAN, A. 2016. Survey of Health, Ageing and Retirement in Europe (SHARE) Wave 4. Release version: 6.1.1. SHARE-ERIC. Disponible desde internet en: http://www.share-project.org/data-documentation/wavesoverview/wave-4.html (con acceso 10/07/2018).

7. CAMINA-MARTÍN, M.; DE MATEO-SILLERAS, B.; MALAFARINA, V.; LOPEZ-MONGIL, R.; NIÑOMARTÍN, V.; LÓPEZ-TRIGO, J.; REDONDO-DELRÍO, M. 2016. Valoración del estado nutricional en Geriatría: declaración de consenso del Grupo de Nutrición de la Sociedad Española de Geriatría y Gerontología. Rev. Española de Geriatría y Gerontología. 51(1):52-57. https:/ / doi.org/10.1016/j.regg.2015.07.007

8. CASTELL, M.; SÁNCHEZ, M.; JULIÁN, R.; QUEIPO, R.; MARTÍN, S.; OTERO, Á. 2013. Frailty prevalence and slow walking speed in persons age 65 and older: implications for primary care. BMC Family Practice. 14:86-86.

https://doi.org/10.1186/1471-2296-14-86

9. CUOCO, A.; CALLAHAN, D.M.; SAYERS, S.; FRONTERA, WR.; BEAN, J.; FIELDING, R. 2004. Impact of Muscle Power and Force on Gait Speed in Disabled Older Men and Women. The Journals of Gerontology. 59(11):1200-1206. https://doi.org/10.1093/gerona/59.11.1200

10. DELMONICO, M.; ZMUDA, J.; TAYLOR, C.; CAULEY, J.; HARRIS, T.; MANINI, T.; NEWMAN, A. 2008. Association of the ACTN3 genotype and physical functioning with age in older adults. J. Gerontol. A Biol. Sci. Med. Sci. 63(11):1227-1234.

11. DEN OUDEN, M.; SCHUURMANS, M.; ARTS, I.; VAN DER SCHOUW, Y. 2011. Physical performance characteristics related to disability in older persons: a systematic review. Maturitas. 69(3):208-219.

https://doi.org/10.1016/j.maturitas.2011.04.008

12. DEPARTAMENTO ADMINISTRATIVO NACIONAL DE ESTADÍSTICA, DANE. 2005. Boletin demográfico No 72 Ed. Celade. Bogota (Colombia). 76p.

13. FEDESARROLLO; FUNDACIÓN SALDARRIAGA CONCHA. 2015. Misión Colombia Envejece: cifras, retos y recomendaciones. Ed. Fundación Saldarriaga Concha (Bogota). 216p.

14. FERNÁNDEZ, J.; HOYOS L. 2020. Efectos de la velocidad de entrenamiento en fuerza sobre diversas manifestaciones de la fuerza en mujeres adultas mayores. Retos. 38:325-332.

15. HAIRI, N.; CUMMING, R.; NAGANATHAN, V.; HANDELSMAN, D.; LE COUTEUR,D.; CREASEY, H.; SAMBROOK, P. 2010. Loss of muscle strength, mass (sarcopenia), and quality (specific force) and its relationship with functional limitation and physical disability: the Concord Health and Ageing in Men Project. J. Am. Geriatr. Soc. 58(11):2055-2062. https://doi.org/10.1111/j.1532-5415.2010.03145.x

16. HARDY, R.; COOPER, R.; AIHIE SAYER, A.; BENSHLOMO, Y.; COOPER, C.; DEARY, I.; KUH, D. 2013. Body mass index, muscle strength and physical performance in older adults from eight cohort studies: the HALCyon programme. PLoSOne. 8(2):e56483. https://doi.org/10.1371/journal.pone.0056483 
17. KIRKWOOD, T. 2017. Why and how are we living longer? Exp Physiol. 102(9):1067-1074. https://doi.org/10.1113/ep086205

18. LOWRY, K.; VALLEJO, A.; STUDENSKI, S. 2011. Successful aging as a continuum of functional independence: lessons from physical disability models of aging. Aging and disease. 3(1):5-15.

19. MAGYARI, P.; LITE, R.; KILPATRICK, J. 2018. ACSM's Resources for the Exercise Physiologist - A Practical Guide for the Health Fitness Professional. Philadelphia: Wolters Kluwer Health. 195p.

20. MARQUES, M.; IZQUIERDO, M.; PEREIRA, A. 2013. Highspeed resistance training in elderly people: A new approach toward counteracting age-related functional capacity loss. Strength and Conditioning J. 35(2):23-29.

21. MCEVOY, B.; VISSCHER, P. 2009. Genetics of human height. Economics \& Human Biology. 7(3):294-306. https://doi.org/10.1016/j.ehb.2009.09.005

22. MIDDLETON, A.; FRITZ, S.; LUSARDI, M. 2015. Walking speed: The functional vital sign. J. Aging Phys. Act. 23(2):314-322. https://doi.org/10.1123/japa.2013-0236

23. MINISTERIO DE SALUD Y PROTECCIÓN SOCIAL. 1993. Resolución número 8430 de 1993. Ministerio de salud y protección social Octubre 4 de 1993. Disponible desde internet en: https: / / www.minsalud.gov.co./sites/rid/Lists/.../ RESOLUCION-8430-DE-1993.PDF (con acceso 4/05/2018).

24. MOY, F.; CHANG, E.; KEE, K. 2011. Predictors of Handgrip Strength among the Free Living Elderly in Rural Pahang, Malaysia. Iranian J. Public Health. 40(4):44-53.

25. MULLEN, S.; MCAULEY, E.; SATARIANO, W.; KEALEY, M.; PROHASKA, T. 2012. Physical activity and functional limitations in older adults: the influence of self-efficacy and functional performance. The Journals of Gerontology Series B: Psychological Sciences and Social Sciences. 67B(3):354-361.

https://doi.org/10.1093/geronb/gbs036

26. NOFUJI, Y.; SHINKAI, S.; TANIGUCHI, Y.; AMANO, H.; NISHI, M.; MURAYAMA, H.; SUZUKI, T. 2016. Associations of walking speed, grip strength, and standing balance with total andcause-specific mortality in a general population of japanese elders. J. Am. Med. Directors Association. 17(2):184.e1-184.e7. https//doi.org/10.1016/j.jamda.2015.11.003
27. OBSERVATORIO DEMOGRÁFICO DE AMÉRICA LATINA. 2011. Envejecimiento poblacional. Disponible desde Internet en:

https://www.cepal.org/en/publications / 7116 observatorio-demografico-america-latina-2011envejecimiento-poblacional (con acceso 21/11/2019).

28. ONG, H.; ABDIN, E.; CHUA, B.; ZHANG, Y.; SEOW, E.; VAINGANKAR, J.; SUBRAMANIAM, M. 2017. Hand-grip strength among older adults in Singapore: a comparison with international norms and associative factors. BMC Geriatrics. 17(1):176. https://doi.org/10.1186/s12877-017-0565-6

29. ORGANIZACIÓN MUNDIAL DE LA SALUD. 2015. Informe mundial sobre el envejecimiento y la salud, Vol. 1. Ed. Organización Mundial de la Salud (Ginebra). 156p.

30. PEEL, N.; KUYS, S.; KLEIN, K. 2013. Gait speed as a measure in geriatric assessment in clinical settings: a systematic review. J. Gerontol. A Biol. Sci. Med. Sci. 68(1):39-46. https://doi.org/10.1093/gerona/gls174

31. PRASITSIRIPHON, O.; POTHISIRI, W. 2018. Associations of grip strength and change in grip strength with all-cause and cardiovascular mortality in a european older population. Clin. Med. Insights Cardiol. 12:1-10. https://doi.org/10.1177/1179546818771894

32. PUTHOFF, M.; NIELSEN, D. 2007. Relationships amongimpairments in lower-extremity strength and power, functional limitations, and disability in older adults. Phys Ther. 87(10):1334-1347. https://doi.org/10.2522/ptj.20060176

33. RAMÍREZ-CAMPILLO, R.; CASTILLO, A.; DE LA FUENTE, C.; CAMPOS-JARA, C.; ANDRADE, D.; ÁLVAREZ, C.; IZQUIERDO, M. 2014. High-speed resistance training ismore effective than low-speed resistance training to increase functional capacity and muscle performance in older women. Exp. Gerontol. 58:51-57. https://doi.org/10.1016/j.exger.2014.07.001

34. REJESKI, W.; MIHALKO, S. 2001. Physical activity and quality of life in older adults. J. Gerontol. A Biol. Sci. Med. Sci. 56(2):23-35.

35. RIKLI, R.; JONES, C. 2013. Development and validation of criterion-referenced clinically relevant fitness standards for maintaining physical independence in later years. Gerontologist. 53(2):255-267. https://doi.org/10.1093/geront/gns071

36. SALLINEN, J.; STENHOLM, S.; RANTANEN, T.; HELIÖVAARA, M.; SAINIO, P.; KOSKINEN, S. 2010 
Hand-Grip strength cut-points to screen older persons at risk for mobility limitation. J. Am. Geriatrics Soc. 58(9):1721-1726.

https://doi.org/10.1111/j.1532-5415.2010.03035.x

37. SANCHEZ-MEDINA, L.; GONZALEZ-BADILLO, J.; PEREZ, C.; PALLARES, J. 2014. Velocity- and power-load relationships of the bench pull vs. bench press exercises. Int. J. Sports Med. 35(3):209-216.

https://doi.org/10.1055/s-0033-1351252

38. SAYERS, S.; GIBSON, K. 2014. High-speed power training in older adults: a shift of the external resistance at which peak power is produced. J. Strength Cond. Res. 28(3):616-621. https://doi.org/10.1519/JSC.0b013e3182a361b8

39. SEINO, S.; SHINKAI, S.; FUJIWARA, Y.; OBUCHI, S.; YOSHIDA, H.; HIRANO, H.; KIN, H.; ISHIZAKI, T.; TAKAHASHI, R. 2014. Reference values and age and sex differences in physical performance measures for community-dwelling older japanese : A pooled analysis of six cohort studies. PLoS ONE. 9(6):e99487.

https://doi.org/10.1371/journal.pone.0099487
40. STUDENSKI, S.;PERERA, S.; PATEL, K.; ROSANO, C.; FAULKNER, K.; INZITARI, M.; GURALNIK, J. 2011. Gait speed and survival in older adults. Jama. 305(1):50-58. https://doi.org/10.1001/jama.2010.1923

41. VERONESE, N.; STUBBS, B.; FONTANA, L.; TREVISAN, C.; BOLZETTA, F.; RUI, M.; SERGI, G. 2017. A Comparison of Objective Physical Performance Tests and Future Mortality in the Elderly People. J. Gerontol. A Biol. Sci. Med. Sci. 72(3):362-368.

https://doi.org/10.1093/gerona/glw139

42. WORLD HEALTH ORGANIZATION. 2015. Study on global AGEing and adult health (SAGE) Geneva: World Health Organization. Disponible desde internet en:

http://www.who.int/healthinfo/sage/en/ (con acceso 10/08/2018).

43. WU, Y.; WANG, W.; LIU, T.; ZHANG, D. 2017. Association of grip strength with risk of all-cause mortality, cardiovasculardiseases, and cancer in community-dwelling populations: A meta-analysis of prospective cohort studies. J. Am. Med. Dir. Assoc. 18(6):551.e17-551.e35. https://doi.org/10.1016/j.jamda.2017.03.011 